\title{
Recombinant interleukin-2 stimulates lymphocyte recovery in patients with severe COVID-19
}

\author{
MENG-EN ZHU, QIAN WANG, SHAOQIONG ZHOU, BIN WANG, LI KE and PING HE \\ Department of Geriatrics, Union Hospital, Tongji Medical College, \\ Huazhong University of Science and Technology, Wuhan, Hubei 430022, P.R. China
}

Received September 9, 2020; Accepted December 15, 2020

DOI: $10.3892 /$ etm.2021.9658

\begin{abstract}
A recently identified type of pneumonia, referred to as coronavirus disease 2019 (COVID-19), which is caused by severe acute respiratory syndrome coronavirus- 2 , has rapidly spread worldwide. Lymphopenia and a proinflammatory cytokine storm frequently occur in patients with severe COVID-19. However, to the best of our knowledge, no specific immunomodulatory therapy for COVID-19 has been reported to date. In the present retrospective case-control study, the potential therapeutic effect of recombinant human interleukin-2 (rIL-2) in patients with severe COVID-19 was demonstrated. A total of 59 patients with severe COVID-19 were admitted to the Union Hospital of Tongji Medical College (Wuhan, China) between 29th January 2020 and 29th February 2020 and were included in the present study. In total, 20 patients received subcutaneous injection of rIL-2 (1 million IU per day) for 7-10 days in addition to regular treatment and were classified as the rIL-2 group. Furthermore, 20 of the 39 patients receiving regular treatment, without the intervention of rIL-2, were matched as the control group. Patients in these two groups were subjected to propensity score matching in terms of clinical characteristics such as age, sex, symptoms, signs, laboratory data and comorbidities. Changes in the lymphocyte count, as well as IL-6 and C-reactive protein (CRP) levels, were analyzed at the time of admission and discharge and any differences between the rIL-2 and non-rIL-2 groups were determined. The results demonstrated an increase in the lymphocyte count and a decrease in CRP levels in the rIL-2 group compared with that in the non-rIL-2 group. The difference in the change of the lymphocyte count between the rIL-2 group and non-rIL-2 group was statistically significant $(\mathrm{P}<0.01)$. Although CRP levels were decreased to a greater extent in the rIL-2 group,
\end{abstract}

Correspondence to: Dr Ping He, Department of Geriatrics, Union Hospital, Tongji Medical College, Huazhong University of Science and Technology, 1277 Jiefang Avenue, Wuhan, Hubei 430022, P.R. China E-mail:pinghe@hust.edu.cn

Key words: COVID-19, recombinant human interleukin-2, lymphopenia, cy tokine, $\mathrm{C}$-reactive protein the difference between the two groups was not statistically significant $(\mathrm{P}>0.05)$. Collectively, the present results suggested that administration of rIL-2 may be a prospective adjuvant therapy for patients with severe COVID-19 and its effects may be mediated by increasing lymphocyte numbers.

\section{Introduction}

Coronavirus disease 2019 (COVID-19), caused by severe acute respiratory syndrome coronavirus-2 (SARS-CoV-2), has been spreading worldwide and poses a critical threat to global health (1). During the COVID-19 pandemic, a previous study has reported the importance and the benefits of maintaining an exercise routine for the health of the population (2). The mechanisms underlying this enhancement of human health may rely on the improvement of immune function, which helps resolve viral-induced respiratory infections. In the early stage of COVID-19, patients usually exhibit a normal or lowered white blood cell (WBC) count in the peripheral blood, along with a marked decrease in the lymphocyte count and an increase in C-reactive protein (CRP) levels. In the advanced stage of COVID-19, numerous inflammatory cytokines, such as interleukins (ILs), TNF and chemokines, are released and attack the immune organs and tissues (3-5). These factors induce an inflammatory storm, leading to disease progression and multiple organ failure. Lymphopenia and the presence of a proinflammatory cytokine storm occur more frequently in patients with severe COVID-19 compared with mild cases and are associated with disease severity (6).

Recently, it was reported that $\mathrm{CD}^{+} \mathrm{T}$-cell numbers are markedly decreased in patients in the early recovery stage of COVID-19. Furthermore, it has been revealed that IL-2, IL-18, IL-4 and TNF superfamily member 13 may be beneficial for the recovery of patients with COVID-19 (7). However, the roles that these cytokines may serve in the therapy of COVID-19 are yet to be elucidated.

The present study reported on the application of recombinant human IL-2 (rIL-2), an immunomodulator commonly used in the treatment of cancer, to the therapy of COVID-19 and investigated the changes in peripheral blood WBC count, lymphocyte count and the levels of IL-6 and CRP. Furthermore, the side effects of rIL-2 were observed simultaneously. The present results may help determine the efficacy and safety of rIL-2 for the treatment of COVID-19. 


\section{Materials and methods}

Study design and participants. The present study was a retrospective single-center case-control study, which included 59 cases of severe COVID-19. The patients were admitted to the West Campus of Union Hospital, Tongji Medical College of Huazhong University of Science and Technology (Wuhan, China) between 29th January 2020 and 29th February 2020. COVID-19 was diagnosed according to the epidemiological and clinical criteria, chest CT scan and lymphocyte count and was confirmed via reverse transcription PCR (RT-PCR) detection of routine pharyngeal swab specimens according to the New Coronavirus Pneumonia Prevention and Control Program (6th edition) published by the National Health Commission of China (8). The final date of follow-up was 15th April 2020.

Severe cases in adults were diagnosed when any of the following criteria were met: i) Respiratory distress ( $\geq 30$ breaths/min); ii) oxygen saturation, $\leq 93 \%$ at rest; and iii) arterial partial pressure of oxygen/fraction of inspired oxygen of $\leq 300 \mathrm{mmHg}(1 \mathrm{mmHg}=0.133 \mathrm{kPa})$. Patients who received subcutaneous injection of rIL-2 (1 million IU per day; production batch no. 20190915; Beijing Sihuan Pharmaceutical Holdings Group Ltd.) with treatment for 7-10 days during hospitalization were classified as the rIL-2 group. Patients who did not receive any rIL-2 during hospitalization were classified as the non-rIL-2 group. Propensity score-matched cohorts were established based on variables that were expected to be potential confounders associated with exposure to rIL-2, including age, sex, fever, fatigue, cough, comorbidities (hypertension, coronary heart disease, diabetes and cerebrovascular disease) and in-hospital medications. The rIL-2 and non-rIL-2 groups were subjected to propensity score matching according to the clinical characteristics, which mainly included sex (high proportion of females in control group), age (high proportion of young subjects in the control group) and comorbidities. As a result of the analysis of the baseline characteristics, there were no significant differences regarding the aforementioned clinical characteristics between the rIL-2 and non-rIL-2 groups before and after matching.

Specific inclusion and exclusion criteria were used to select the study population. The inclusion criteria were as follows: i) Patients admitted to the hospital with severe COVID-19; and ii) age $>18$ years. The exclusion criteria were as follows: i) Incomplete medical records (e.g., transfer to another hospital); ii) pregnancy; iii) patients who did not survive during hospitalization; iv) recurring fever; v) severe hepatic dysfunction; and vi) severe renal dysfunction. The study protocols were approved and informed consent from the patients was waived by the ethics committee of Union Hospital, Tongji Medical College Huazhong University of Science and Technology (Wuhan, China).

Data collection. In the present study, the admission and discharge data of patients in the rIL-2 and non-rIL-2 groups regarding demographic information, clinical characteristics, laboratory data, presence of comorbidities and therapeutic interventions during the hospitalization were collected. The demographic information (age and sex) and clinical characteristics (fever, cough and fatigue) of the patients were collected from an electronic medical system. Laboratory data (blood cell count, IL-6, CRP, hepatic function and renal function) were collected from a laboratory information system. Comorbidities (hypertension, coronary heart disease, diabetes and cerebrovascular diseases) were extracted from the patients' medical history records. Information on in-hospital medications and interventions was collected from the records of the treating clinicians. Information regarding the length of hospital stay was collected at the time of patient discharge from the hospital. Any identifying personal information, such as name and ID, was anonymized and each participant was assigned a study ID using an electronic coding system prior to data extraction to preserve patient anonymity. Data were carefully reviewed and confirmed by experienced physicians and were checked to guarantee the accuracy of the data extraction procedures.

Statistical analysis. Statistical significance of differences in parameters within the same group of patients prior to and after treatment were analyzed using Wilcoxon's signed-rank test. A Mann-Whitney U test or $\chi^{2}$ test was performed to compare differences between the groups with or without treatment of rIL-2. Continuous variables were presented as the median [interquartile range (IQR)] and categorical variables were expressed as $\mathrm{n}(\%)$. A two-sided $\mathrm{P}<0.05$ was considered to indicate a statistically significant difference. Data were analyzed using SPSS version 23.0 (IBM Corp.).

\section{Results}

Participants. Between the 29th January and 29th February 2020, 72 adult patients with COVID-19 were hospitalized at the Union Hospital, Tongji Medical College Huazhong University of Science and Technology (Wuhan, China). After excluding 5 patients who succumbed to the disease, 3 who were transferred to other hospitals, 2 with recurring fever, 1 with severe hepatic dysfunction and 2 with severe renal dysfunction, a total of 59 patients were included in the present study. A total of 20 patients received rIL-2 treatment. From the 39 patients in the non-rIL-2 group, 20 patients were selected and paired with the rIL-2 group (Fig. 1).

Baseline characteristics of the participants and comparisons between groups. As presented in Table I, the median age of all patients was 56 years [interquartile range (IQR), 48-64 years], ranging from 23 to 88 years. Of all of the patients, $54.24 \%$ were male. Comorbidities such as hypertension $(28.81 \%)$, diabetes $(15.25 \%)$, coronary heart disease $(6.78 \%)$ and cerebrovascular disease $(10.17 \%)$ were common. The symptom of fatigue was observed in $49.15 \%$ patients prior to treatment and the symptom of cough (77.97\%) was observed in most patients before treatment. Furthermore, $69.49 \%$ of the patients had a fever on admission. To observe the drug-associated adverse effects, the patients' body temperature in the rIL-2 group was maintained under control (axillary temperature, $36-37^{\circ} \mathrm{C}$ ) prior to rIL-2 treatment in the present study. The median WBC and neutrophil counts in patients were within the normal ranges, 5.900 $\times 10^{9} / 1$ (IQR, 4.2,7.2) and 3.980x $10^{9} / 1(2.8,5.1)$, respectively (normal

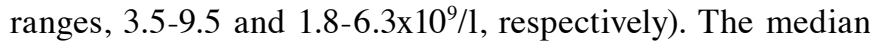
lymphocyte count decreased to $0.980 \times 109 / 1(0.8,1.4)$, which was lower than the lower limit of the normal range (normal range, 1.1-3.2x $\left.10^{9} / 1\right)$. The levels of serum IL-6 and CRP were increased to $6.420 \mathrm{pg} / 1(1.5,16.2)$ and $27.220 \mathrm{mg} / 1(4.7,48.2)$, respectively (the upper limit of the normal ranges: IL-6, $<1.5 \mathrm{pg} / \mathrm{l}$; CRP, 


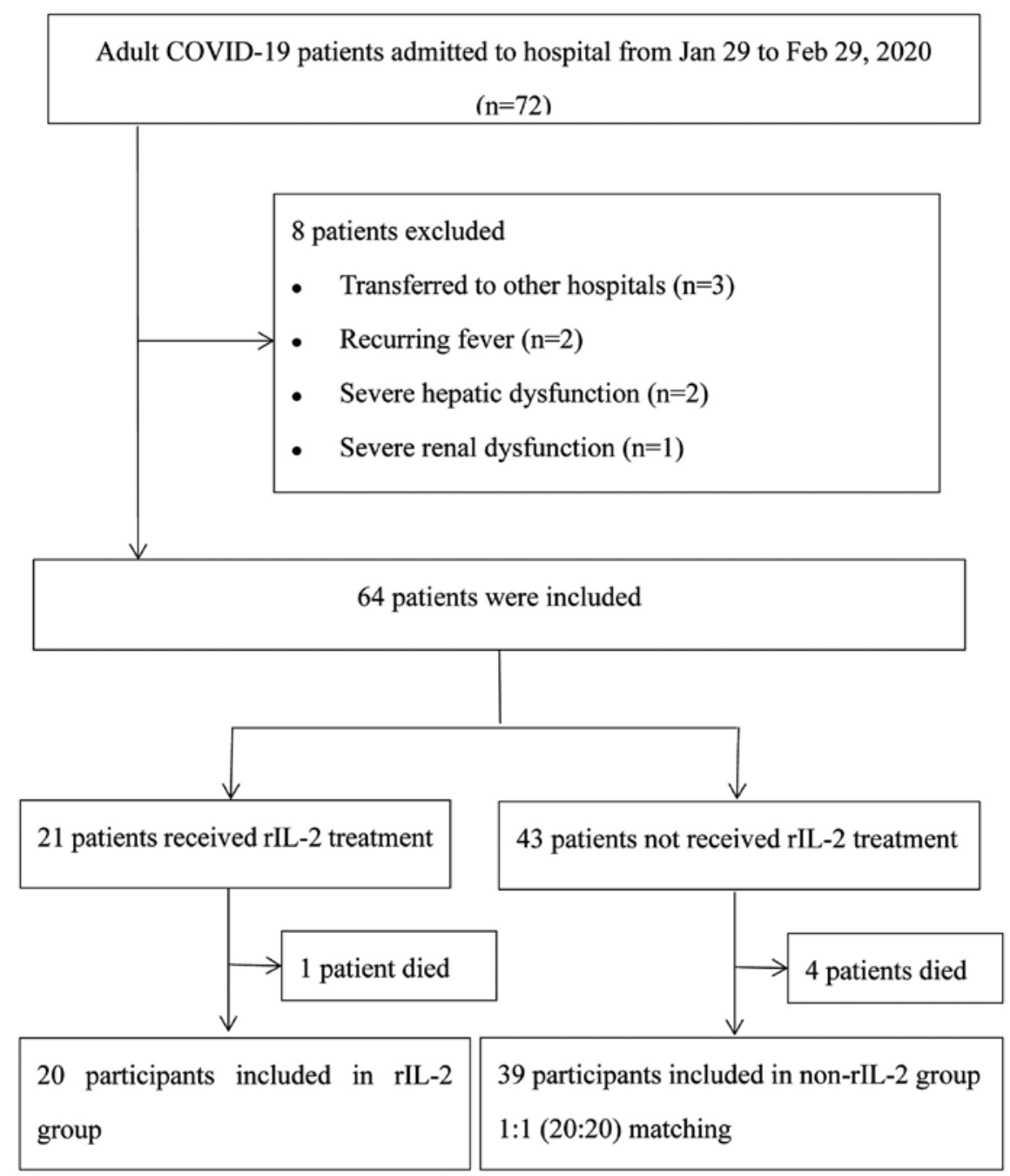

Figure 1. Patient flowchart. rIL-2, recombinant human interleukin-2; COVID-19, coronavirus disease 2019.

$<8 \mathrm{mg} / \mathrm{l})$. During hospitalization, $53(89.83 \%)$ of the patients received antiviral drug therapy, $44(74.58 \%)$ received antibiotic therapy, $5(10.17 \%)$ received systemic corticosteroid therapy and $56(94.92 \%)$ received Traditional Chinese Medicine therapy, specifically Lianhua Qingwen Zhiji (Forsythiae and Honeysuckle Flower Pestilence-Clearing Preparations) (9). The aforementioned clinical characteristics in both groups prior to and after matching did not exhibit any significant differences (Table I).

Comparison of laboratory results in the rIL-2 group on admission and at discharge. In the rIL-2 group, the following variables exhibited a statistically significant difference (all $\mathrm{P}<0.05$ ) at discharge vs. the baseline: Lymphocyte count, neutrophil count, IL-6 levels, CRP levels, blood urea nitrogen (BUN) and serum creatinine (Scr) levels. The lymphocyte count was significantly higher than that at discharge vs. the baseline in the rIL-2 group $(\mathrm{P}<0.01)$, whilst the neutrophil count, CRP and IL-6 levels were significantly decreased at discharge (all $\mathrm{P}<0.05)$. The WBC count in the rIL-2 group at discharge was similar compared with that on admission $(\mathrm{P}>0.05)$. Of note, rIL-2 treatment had an effect on renal function, as it decreased BUN levels and Scr levels $(\mathrm{P}<0.05)$. However, there were no significant differences in hemoglobin $(\mathrm{Hb})$ levels, platelet (PLT) count, alanine aminotransferase (ALT) levels or aspartate aminotransferase (AST) levels at discharge vs. the baseline in the rIL-2 group (all $\mathrm{P}>0.05$; Table II).

Comparison of laboratory results in the non-rIL-2 group on admission and at discharge. A significant increase in the lymphocyte count $(\mathrm{P}<0.01)$ was noted at discharge vs. the baseline in the non-rIL-2 group receiving regular treatment. Furthermore, IL- 6 and CRP levels exhibited a significant decrease after regular treatment $(\mathrm{P}<0.05)$. No obvious changes in the WBC and neutrophil count were identified (all $\mathrm{P}>0.05$ ). In addition, regular treatment had no influence on $\mathrm{Hb}$ levels, PLT count, ALT, AST, BUN or Scr levels (all P>0.05; Table II).

Comparison of laboratory results between the rIL-2 and non-rIL-2 groups at discharge. Compared to the non-IL-2 group, the rIL-2 group had a significantly higher level of lymphocytes at discharge $(\mathrm{P}<0.01)$. However, there were no significant differences in the WBC count, neutrophil count, CRP levels and IL-6 levels between the two groups (all P>0.05). Furthermore, rIL-2 treatment in addition to regular treatment did not significantly alter Hb levels, PLT count, ALT, AST, BUN or Scr levels (vs. non-rIL-2 group with regular treatment, $\mathrm{P}>0.05$; Table II). There were also no differences in the length of hospital stay and the 


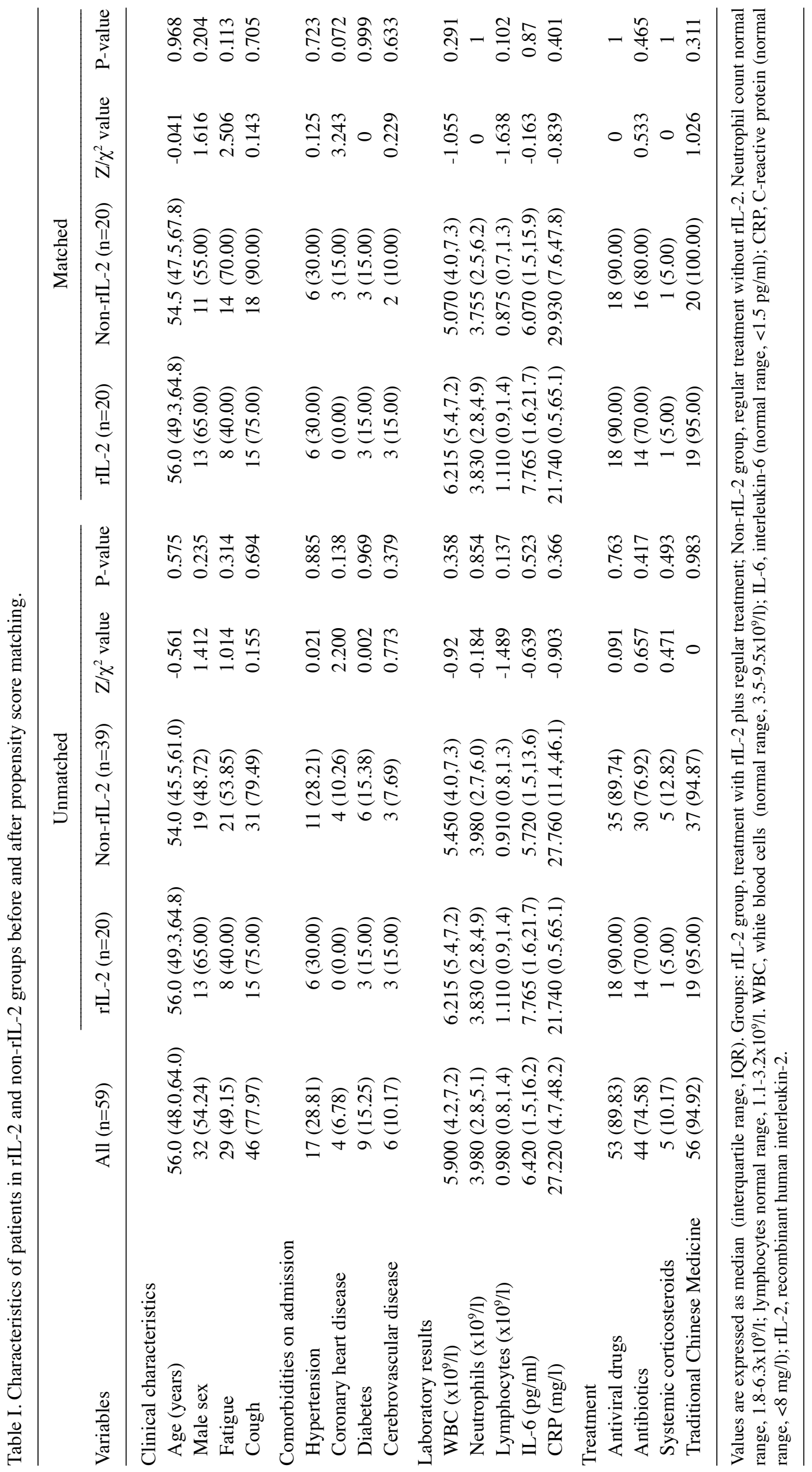




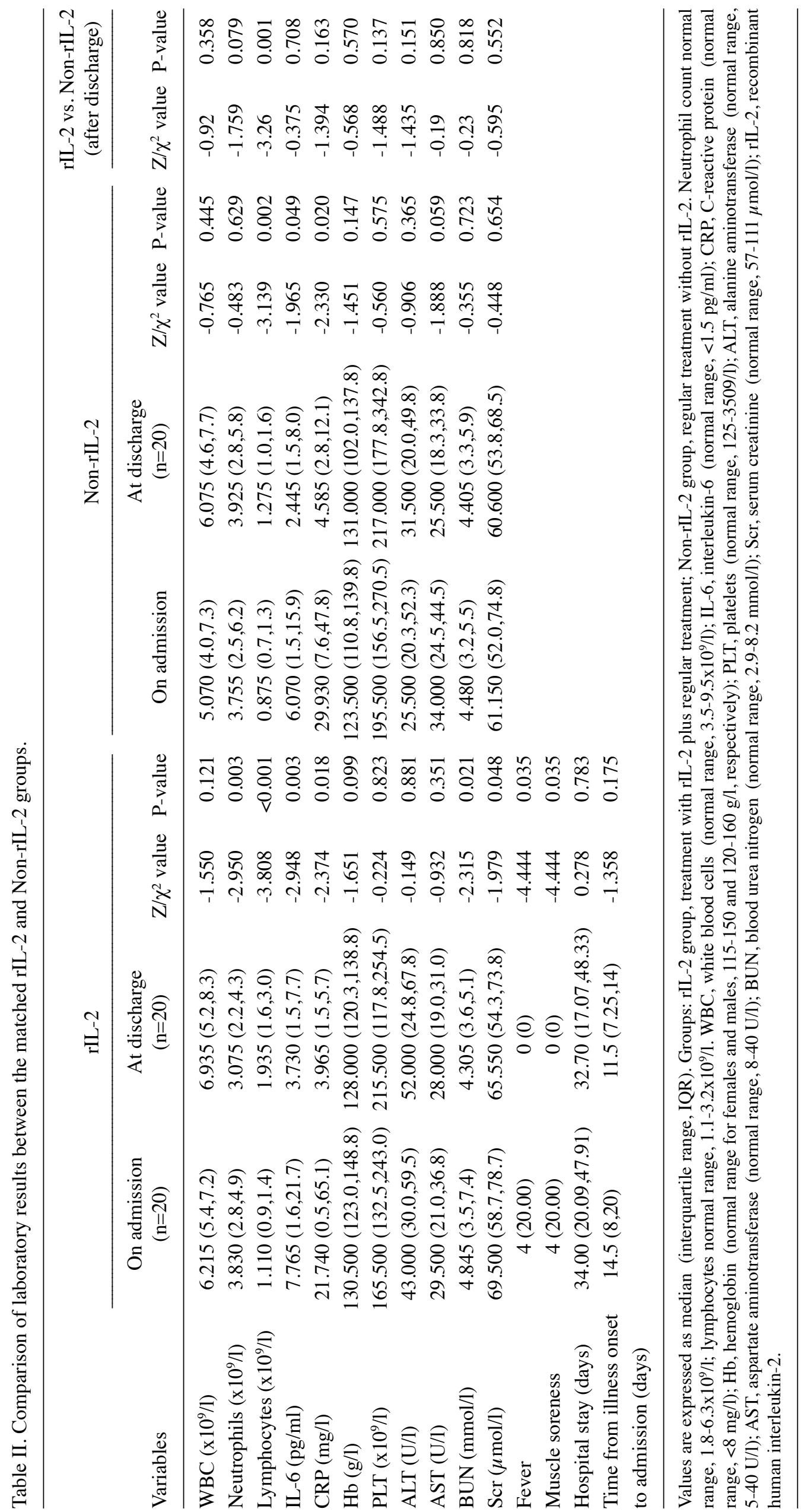


time from illness onset to admission between the two groups ( $\mathrm{P}>0.05$; Table II).

Drug-associated adverse effects of rIL-2 treatment. After regular treatment, including antiviral drugs, antibiotics, corticosteroids and Traditional Chinese Medicine, all participants had a normal body temperature (axillary temperature, $36-37^{\circ} \mathrm{C}$ ). In the rIL-2 group, 4 (20\%) cases of fever associated with rIL-2 treatment (10) were reported, with a high body temperature $\left(37^{\circ} \mathrm{C} \leq \mathrm{T} \leq 38.5^{\circ} \mathrm{C}\right)$ for $<4$ days. These patients complained of muscle soreness accompanied with a fever. No patient with new onset of fever was reported in the non-rIL-2 group. The differences regarding fever and muscle soreness between the rIL-2 and non-rIL-2 groups were significant (both $\mathrm{P}<0.05$; Table II).

\section{Discussion}

Recent studies have reported that lymphopenia is one of the typical laboratory abnormalities of COVID-19 $(6-8,11,12)$. Lymphopenia was observed in $44.4 \%$ (12/27) of mild cases and in $84.6 \%(11 / 13)$ of severe cases at the onset of COVID-19. The absolute counts of lymphocytes in the peripheral blood of the severe cases were significantly decreased at the time-point of disease onset and these still further decreased after 4-6 days. At 7-15 days after disease onset, the lymphocyte counts gradually increased in severe cases and reached a comparable level to those of mild cases at 16 days after disease onset. Sustained decreases in separate $\mathrm{CD}^{+} \mathrm{T}$-cell, $\mathrm{CD}^{+} \mathrm{T}$-cell and $\mathrm{CD} 4^{+} \mathrm{T}$-cell counts were also observed in severe cases (6). $\mathrm{CD} 4^{+} \mathrm{T}$ cells were markedly decreased in patients in the early recovery stage of COVID-19. $\mathrm{T}$ - and B-cell clones have also been reported to be enhanced during the recovery stage of COVID-19 (7). Accumulating evidence from cohort studies and clinical trials has revealed that higher $\mathrm{CD}^{+} \mathrm{T}$-cell counts are associated with reductions in morbidity and mortality from both acquired immunodeficiency syndrome (AIDS) and serious non-AIDS conditions, including cardiovascular diseases (13-15). All of these observations suggest that increasing the lymphocyte count, particularly that of $\mathrm{CD}^{+} \mathrm{T}$ cells, may be a prospective therapy for COVID-19.

In previous studies and clinical trials, IL-2, which is mainly produced by $\mathrm{CD} 4^{+} \mathrm{T}$ cells and $\mathrm{CD} 8^{+} \mathrm{T}$ cells, has acted as a T-cell activator (14) rIL-2 has been demonstrated to be an effective method for enhancing the efficacy of antitumor immunotherapy (15). Several studies have reported that rIL-2 exerts an immunomodulatory effect in viral infections. An in vivo study using an immunocompetent $\mathrm{BALB} / \mathrm{c}$ mouse model revealed that repeated application of rIL-2 significantly enhanced the antiviral effect of the low number of lymphocytes in several tissues, both in prophylaxis and in therapy (16). In a prospective cohort study on patients with HIV, CD4 ${ }^{+}$T-cell counts were observed to increase after rIL-2 treatment (17). Furthermore, a low dose of rIL-2 selectively modulated CD $4^{+}$ T-cell subsets in patients with systemic lupus erythematosus and markedly reduced the activity of the disease (18).

The presentresults demonstrated that IL-2 was able toincrease lymphocyte numbers in the peripheral blood. Furthermore, IL-6 and CRP levels were significantly decreased after rIL-2 treatment compared with those prior to rIL-2 treatment. However, the differences among the neutrophil numbers, Hb, CRP and IL-6 levels between the rIL-2 and the non-rIL-2 groups were not significant $(\mathrm{P}>0.05)$. It also indicated that the length of hospital stay was not affected by rIL-2. The criteria for patient discharge were the remission of clinical symptoms (including fever, cough, muscle soreness and fatigue), recovery on chest CT and twice negative RT-PCR detection for SARS-CoV-2 (the interval of the two pharyngeal swab specimens was over $24 \mathrm{~h}$ ). In addition, the abnormal laboratory data turning to normal, recovery of patients' physical strength, their psychological health and control of comorbidities were also taken into account when making a decision regarding discharge of the patients of the present study. On the one hand, the length of hospitalization was considered when deciding on patient discharge. On the other hand, the aforementioned factors influenced the length of hospitalization. Therefore, the length of hospital stay reflected the overall situation of the patients recovering from the disease and not only the therapeutic effect of rIL-2. Recently, it has been reported that $\mathrm{T}$ cell-produced IL-2 promotes the survival, proliferation, differentiation and antibody production of $\mathrm{B}$ cells in patients with COVID-19. Consequently, B cells produce numerous SARS-COV-2-specific antibodies to clear the virus $(7,19)$. A study has indicated that the low levels of IL-2 in plasma may be a warning factor of disease deterioration in patients with severe COVID-19, which may lead to a significant decrease of $\mathrm{CD}^{+} \mathrm{T}$ cells and lymphocytes (14). Therefore, suitable provision of IL-2 may support the remission and recovery of patients with COVID-19 by exerting immunoregulatory effects (14). In the present study, additional administration of rIL-2 to patients with COVID-19, which stimulates lymphocyte recovery, was indicated to have potential therapeutic effects. For instance, the increased number of lymphocytes may be associated with changes of T-cell subsets, particularly the increased number of $\mathrm{CD}^{+} \mathrm{T}$ cells. In addition, rIL-2 may mimic the effect of native IL-2 and stimulate the survival, proliferation, differentiation and antibody production of $\mathrm{B}$ cells, which accelerates the clearance of the virus. Furthermore, rIL-2 may relieve the inflammatory storm by decreasing the levels of cytokines (20-22). However, the precise mechanisms have remained to be elucidated.

In the present study, patients who succumbed to COVID-19 were excluded, because they were unable to provide complete clinical data and were associated with confounding factors, including rapid disease progression, patients were too weak to state their condition due to exacerbation of the disease and cause of death was not because of COVID-19, all which adversely affected analysis. rIL-2 was well-tolerated by patients with severe COVID-19. Only four cases of adverse events were reported in the rIL-2 group. The adverse reactions included fever $\left(37^{\circ} \mathrm{C} \leq \mathrm{T} \leq 38.5^{\circ} \mathrm{C}\right)$ and muscle soreness. All adverse events reported were mild, transient and completely relieved after stopping rIL-2 treatment. In addition, no abnormal changes in laboratory measurements were clinically significant or considered to be associated with rIL-2.

There were certain limitations to the present study. First, it was a retrospective single-center case-control study. The hospital was assigned by the government to accept mostly patients with severe and critical COVID-19. Therefore, only patients with severe COVID-19 were included in the present study, which may cause selection bias. The results of the present study were also limited by the small size of the cohort and the short duration of rIL-2 treatment. Furthermore, the effects of different dosages of rIL-2 in different stages of COVID-19 were 
not investigated. The changes in cellular and humoral immune functions, complement and cytokines prior to and after administration of rIL-2 were also not investigated in the present study.

In conclusion, to the best of our knowledge, the present study was the first to investigate the potential therapeutic effect of rIL-2 in COVID-19. rIL-2 may be used as a prospective therapy in patients with severe COVID-19, as it may accelerate disease recovery by increasing the lymphocyte count.

\section{Acknowledgements}

The authors acknowledge all health-care workers from the Beijing, Hainan and Anhui medical teams involved in the diagnosis and treatment of patients at the Union Hospital, Tongji Medical College Huazhong University of Science and Technology (Wuhan, China).

\section{Funding}

No funding was received.

\section{Availability of data and materials}

The datasets used and/or analyzed during the present study are available from the corresponding author on reasonable request.

\section{Authors' contributions}

PH and MEZ designed study, performed statistical analysis and wrote the manuscript. MEZ, QW, BW, SZ and LK collected data, analyzed data and interpreted references for the paper. MEZ and QW checked and confirmed the authenticity of the raw data. Moreover, all authors participated in the reviewing and revision of this paper critically. All authors read and approved the final version of the manuscript.

\section{Ethics approval and consent to participate}

The study protocols were approved and informed consent from the patients was waived by the ethics committee of Union Hospital, Tongji Medical College Huazhong University of Science and Technology (Wuhan, China).

\section{Patient consent for publication}

Not applicable.

\section{Competing interests}

The authors declare that they have no competing interests.

\section{References}

1. WHO: Coronavirus disease 2019 (COVID-19) Situation report-Weekly epidemiological update - 29 December 2020. Available from: https://www.who.int/publications/m/item/ weekly-epidemiological-update---29-december-2020.

2. Ravalli S and Musumeci G: Coronavirus Outbreak in Italy: Physiological benefits of home-based exercise during pandemic. J Funct Morphol Kinesiol 5: 31, 2020.

3. Dimitriou F, Matter AV, Mangana J, Urosevic-Maiwald M, Micaletto S, Braun RP, French LE and Dummer R: Cytokine release syndrome during sequential treatment with immune checkpoint inhibitors and kinase inhibitors for metastatic melanoma. J Immunother 42: 29-32, 2019.
4. Huang C, Wang Y, Li X, Ren L, Zhao J, Hu Y, Zhang L, Fan G, $\mathrm{Xu}$ J, Gu X, et al: Clinical features of patients infected with 2019 novel coronavirus in Wuhan, China. Lancet 395: 497-506, 2020.

5. Nunnari G, Sanfilippo C, Castrogiovanni P, Imbesi R, Li Volti G, Barbagallo I, Musumeci G and Di Rosa M: Network perturbation analysis in human bronchial epithelial cells following SARS-CoV2 infection. Exp Cell Res 395: 112204, 2020.

6. Liu J, Li S, Liu J, Liang B, Wang X, Wang H, Li W, Tong Q, Yi J, Zhao L, et al: Longitudinal characteristics of lymphocyte responses and cytokine profiles in the peripheral blood of SARS-CoV-2 infected patients. EBioMedicine 55: 102763, 2020.

7. Wen W, Su W, Tang H, Le W, Zhang X, Zheng Y, Liu X, Xie L, $\mathrm{Li}$ J, Ye J, et al: Immune cell profiling of COVID-19 patients in the recovery stage by single-cell sequencing. Cell Discov 6: 31, 2020.

8. Wang D, Hu B, Hu C, Fangfang Zhu F, Liu X, Zhang J, Wang B, Xiang $\mathrm{H}$, Cheng Z, Xiong Y, et al: Clinical characteristics of 138 hospitalized patients with 2019 Novel Coronavirus-infected pneumonia in Wuhan. JAMA 323: 1061-1069, 2020.

9. Ni L, Chen L, Huang X, Han C, Xu J, Zhang H, Luan X, Zhao Y, Xu J, Yuan W, et al: Combating COVID-19 with integrated traditional Chinese and Western medicine in China. Acta Pharm Sin B 10: 1149-1162, 2020.

10. Nagayama H, Takahashi S, Takahashi T, Ogami K, Ikebuchi K, Tojo A, Tani K and Asano S: IL-2LAK therapy for refractory acute monoblastic leukemia relapsing after unrelated allogeneic bone marrow transplantation. Bone Marrow Transplant 23: 183-185, 1999.

11. Chen N, Zhou M, Dong X, Qu J, Gong F, Han Y, Qiu Y, Wang J, Liu Y, Wei Y, et al: Epidemiological and clinical characteristics of 99 cases of 2019 novel coronavirus pneumonia in Wuhan, China: A descriptive study. Lancet 395: 507-513, 2020.

12. Chen G, Wu D, Guo W, Cao Y, Huang D, Wang H, Wang T, Zhang X, Chen $\mathrm{H}, \mathrm{Yu} \mathrm{H}$, et al: Clinical and immunological features of severe and moderate coronavirus disease 2019. JClin Invest 130: 2620-2629, 2020.

13. Pett SL, Kelleher AD and Emery S: Role of interleukin-2 in patients with HIV infection. Drugs 70: 1115-1130, 2010.

14. Shi H, Wang W, Yin J, Ouyang Y, Pang L, Feng Y, Qiao L, Guo X, Shi $\mathrm{H}$, Jin R, et al: The inhibition of IL-2/IL-2R gives rise to $\mathrm{CD} 8^{+}$ $\mathrm{T}$ cell and lymphocyte decrease through JAK1-STAT5 in critical patients with COVID-19 pneumonia. Cell Death Dis 11: 429, 2020.

15. Mahmoudpour SH, Jankowski M, Valerio L, Becker C, Espinola-Klein C, Konstantinides S, Quitzau K and Barco S: Safety of low-dose subcutaneous recombinant interleukin-2: Systematic review and meta-analysis of randomized controlled trials. Sci Rep 9: 7145, 2019.

16. Reddehase MJ, Mutter W and Koszinowski UH: In vivo application of recombinant interleukin 2 in the immunotherapy of established cytomegalovirus infection. J Exp Med 165: 650-656, 1987.

17. Crespo M, Caragol I, Falcó V, Ribera E, Urban S and Pahissa A: Efficacy of recombinant interleukin-2 (rIL-2) in patients with advanced HIV-1 infection and blunted immune response to HAART. Enferm Infecc Microbiol Clin 26: 27-31, 2008.

18. He J, Zhang X, Wei Y, Sun X, Chen Y, Deng J, Jin Y, Gan Y, Hu X, Jia R, et al: Low-dose interleukin-2 treatment selectively modulates CD4(+) T cell subsets in patients with systemic lupus erythematosus. Nat Med 22: 991-993, 2016.

19. Zhou L, Opalinska J, Sohal D, Yu Y, Mo Y, Bhagat T, Abdel-Wahab O, Fazzari M, Figueroa M, Alencar C, et al: Aberrant epigenetic and genetic marks are seen in myelodysplastic leukocytes and reveal Dock4 as a candidate pathogenic gene on chromosome 7q. J Biol Chem 286: 25211-25223, 2011.

20. Schub D, Klemis V, Schneitler S, Mihm J, Lepper PM, Wilkens H, Bals R, Eichler H, Gärtner BC, Becker SL, et al: High levels of SARS-CoV-2-specific T cells with restricted functionality in severe courses of COVID-19. JCI Insight 5: 5, 2020.

21. Sato Y, Keino H, Nakayama M, Kano M and Okada AA: Effect of in vivo expansion of regulatory T cells with IL-2/anti-IL-2 antibody complex plus rapamycin on experimental autoimmune uveoretinitis. Ocul Immunol Inflamm: May 27, 2020 (Epub ahead of print). doi: 10.1080/09273948.2020.1757119.

22. Overwijk WW, Tagliaferri MA and Zalevsky J: Engineering IL-2 to give new life to T cell immunotherapy. Annu Rev Med: Nov 6, 2020 (Epub ahead of print). doi: 10.1146/annurevmed-073118-011031. International (CC BY-NC-ND 4.0) License. 